\title{
ANTROPOLOGIA DI UN INTELLETTUALE, GIUSEPPE CASSIERI
}

\author{
GRAZIA SOTIS \\ (Loyola University Chicago \\ John Felice Rome Center)
}

\begin{abstract}
Abstact
Giuseppe Cassieri's last novel depicts the profound crisis a man of culture lives through his own time: it is society's lack of consideration, loss of humanistic and traditional values, all mirrored in the illness of the protagonist who represents the condition of the intellectual of all times always aware of the need of changes. Cassieri's novel can also be considered an essay where the author analyzes men in relation to theirs own environment and ideas as they are portrayed in their physical and mental conditions.
\end{abstract}

Poetica di un infelice è l'ultima fatica letteraria di Giuseppe Cassieri pubblicata con Manni Editore. L'illustrazione della clessidra, che fa da sfondo al titolo, delinea la condizione dell'uomo di pensiero nel tempo passato, presente e futuro, e del disagio che lui continua a vivere in una società sorda alle necessità di profondi cambiamenti e alla giusta dimensione della narrazione che l'informazione dei talk-show e dei facili consensi hanno fatto perdere di vista. Il personaggio del professor Saverio Lamanna ne è testimone e capro espiatorio, vittima dell'indifferenza e della superficialità che lo circondano. 
Il nuovo romanzo di Cassieri è quindi anche un saggio dove l'autore analizza la figura dello studioso in rapporto al proprio ambiente, rappresentandolo nella sua essenza fisica e mentale, condizione fondamentale di chi ha scelto di vivere la propria vita senza compromessi ed ipocrisie, indagando nella profondità della psiche dell'uomo.

Il professor Lamanna è l'intellettuale che si trova a dover affrontare le barriere insormontabili dell'incomprensione che determinano il suo isolamento e graduale allontanamento dalla realtà e, di conseguenza, giustificano i gesti eclatanti ed esasperati, se non addirittura paranoici, dell'uomo di cultura che, per questo motivo, rasenta la dissociazione del proprio io.

Un precursore della condizione di un simile personaggio è da ricercare nell'antecedente letterario del racconto di Herman Melville, "Bartleby, lo scrivano", dove il lavoro di copista limita la capacità dell'uomo di esprimersi creativamente e l'isolamento fisico circoscritto ad un ufficio, la cui finestra affaccia sui muri degli altri edifici, gli preclude ogni possibilità di evasione e di riscatto da una situazione che lo umilia e che gli fa vivere un disagio sociale trasformatosi poi in paranoia $e$ in asimmetrie di percezioni. Ne scaturisce una profonda mancanza di volontà di agire e di comunicare anche con il suo datore di lavoro, un avvocato che cerca in tutti i modi di interagire con lui. La compostezza stoica di Bartleby si manifesta nel rigore linguistico della affermazione divenuta famosa "I would prefer not to" "Preferirei di no", che tuttavia esprime assenza emotiva ed elusività.

Il professor Lamanna offre le sue capacità creative ed interpretative a studenti che devono scrivere tesi di laurea, da parte sua non esiste un dialogo diretto con il relatore dei lavori, lui non occupa nessun ruolo, perciò diventa figura invisibile di un pensiero che non è in grado di poter difendere. Questo personaggio trova i suoi prodromi culturali negli altri 
studiosi del romanzo Il muro di Gutenberg (del 1999) che si erano riuniti sull'isola di Ventotene per discutere dei temi pertinenti il terzo millennio e del contributo che ognuno, attraverso le rispettive discipline, potesse apportare nella società che s'apriva alla nuova era. Nel recente libro pensieri e suggerimenti confluiscono in questo unico personaggio dimostrando il fallimento di tutti quei tentativi di discussione. Fra i pensatori di Ventotene c'era anche una poetessa, Eva, che eludeva ogni possibilità di confronto a tal punto da respingere ogni tentativo di dialogo per continuare ad essere fedele solo a se stessa: il suicidio della donna è stato il modo di evadere dalla realtà e il gesto di ribellarsi che viene ripreso e descritto nella Poetica di un infelice. Infatti la malattia del professore diventa evasione da momenti di disagio dell'uomo, e se da un lato evidenzia il risvolto degradante di una società che è sorda alle idee, dall'altro simboleggia un atto di rifiuto e, perché no, di autodifesa.

Anche Lamanna soccombe, non per scelta come Eva, ma per la sua malattia che lo degrada e lo umilia. L'epilessia oltre a mostrarlo in tutta la sua fragilità mentale e fisica, agisce da freno inibitorio accentuando il muro fra sé e gli altri. Nel romanzo saggio Cassieri sviluppa ulteriormente il risvolto di quelle lunghe discussioni, avvenute sull'isola di Ventotene, nel delineare la nuova poetica. È quindi legittimo definire questo libro un saggio in virtù del ruolo che il personaggio viene a coprire, con i suoi punti di forza e di debolezza condizionati dal contesto sociale.

A questo punto è bene ricercare nella figura di Willy de $I l$ muro di Gutenberg la voce dello stesso autore, dell'importanza dell'antropologia nella letteratura, essendo lui un antropologo che "[...] non inventa...non conosce rapimenti...rielabora magari impreziosisce vecchi manufatti, toglie crosta, segnala guasti e vizi di forme sotto gli stucchi e li tramanda" (11). 
Il contributo della sua esperienza è volto al recupero della dimensione della storia e della società che altrimenti andrebbe perduta, e la potremmo porre come forza e prova contrastante all'affermazione di Wolf Lepenies quando dice nel suo libro Ascesa e declino degli intellettuali in Europa:

[...] l'arte, che a lungo tempo ha compensato la scomparsa della dimensione magica e mitica del mondo, deve ora fare $\mathrm{i}$ conti con la probabile fine di esso. L'arte ci deve offrire sorprese e alternative, deve ricordarci il passato e convincerci che ci potrà essere un futuro. (12)

Ciò spiega come Il muro di Gutenberg non a caso abbia suscitato l'interesse di studiosi di sociologia: ne è testimonianza l'attenzione datagli da Franco Ferrarotti, da Ulderico Bernardi, e la dedica fatta da Cassieri allo studioso Alfonso di Nola. Su questa linea, Poetica di un infelice diventa un altro tassello interpretativo della funzione del romanzo e della letteratura oggi. Questo rafforza tuttavia la posizione di Lepenies quando sottolinea l'importanza della letteratura, di come essa sia la migliore delle sociologie, alla quale possiamo ora aggiungere la migliore degli studi di antropologia che, con la Poetica di un infelice, analizza il comportamento dell'uomo in rapporto alla cultura del suo tempo, così come lui la vive e la percepisce. Infatti Cassieri presenta questo nuovo personaggio, dalle spiccate capacità interpretative, elusivo e per questa ragione ambiguo. La sua modernità si definisce proprio nella ambiguità dell'esistenza.

Il disagio dell'uomo è narrato con una spettacolarità repressa: infatti, ripassa con la pittura rossa i versi di Leopardi incisi sul marmo che nessun più legge. L'azione del professore diventa un desiderio di visibilità per il messaggio sull' importanza del recupero del passato. Il risultato è paradossale perché, come gli scrittori, poeti, pittori,scultori del Futurismo che istigavano il pubblico ad una presa di coscienza del bisogno di 
cambiamento della loro società e diventavano loro stessi espressioni di accordo con i tempi nuovi, con lo stesso intento Lamanna vuole scuotere le coscienze degli uomini. Gli episodi dell'anno scorso dell'artista che aveva colorato di rosso l'acqua della Fontana di Trevi e poi aveva fatto rotolare dalla scalinata di Trinità dei Monti centinaia di palline colorate, sono gesti di chi è deluso dalla poca o superficiale attenzione che viene data agli uomini di pensiero. Questo tipo di manifestazioni si carica di una valenza antropologica, nel collegare attraverso questi accadimenti il passato con il presente e restituire all'uomo la sua dimensione storicoculturale e riconquistare la giusta visibilità.

Oggi la persona di cultura è invisibile, gli si passa accanto senza accorgersi di lui, a meno che non avvenga un incidente a porlo alla nostra attenzione, come è avvenuto per il direttore della Biblioteca Enzo Castellani che per un tamponamento, nel guardare fuori dal finestrino, è stato attratto da un personaggio che "centellinava le parole commemorative" (6) del piastrino di Ennio Flaiano. Così è descritto da chi lo osserva:

Lo scorgevo in posizione obliqua, un po'curvo, vicinissimo alla lastra. Poi si era voltato di scatto verso il mio parabrezza, quasi avesse percepito la curiosità riflessa che provocava. Magro,..., capelli ricciuti nerissimi, un filo di barba sulle guance scure e, a contrasto, pupille azzurrine di singolare mobilità: sembravano ritrarsi e affacciarsi con fastidio sul mondo. Difficile ipotizzare l'appartenenza. Forse un extracomunitario interessato alla nostra cultura, forse un marmista in transito che esaminava il lavoretto del collega e lo giudicava piatto, seriale, stando all'espressione critica delle labbra. (6) 
Quando il dottor Enzo Castellani lo rivede la seconda volta, che ripassava di vernice rossa i versi di Leopardi dell'Infinito, pensa che possa essere un "intellettuale anarchico ferito dalla società" oppure "uno dei reietti sparsi nella bolgia metropolitana", o una "vittima di erronea giustizia". Di sicuro conclude "qualcuno allenato a pensare e a sconcertare" (9). Anche l'abbigliamento dell'uomo, "avviluppato nella mantella arancione, calzini stinti e sciarpa al collo" (5), lo aveva colpito la prima volta e la descrizione già anticipa il disagio che non tarda a manifestarsi.

A questo punto è bene sottolineare che Cassieri non lascia nulla fuori posto. I suoi referenti culturali, in seguito saranno menzionati Giordano Bruno e Tommaso Campanella, aiutano ad orientare il lettore ad una corretta interpretazione del testo. Essi fanno anche da contrappunto stilistico a momenti di azione e di stasi della scrittura e del pensiero di Lamanna.

Stasi e immobilità, incapacità di agire, di fare, di muoversi come già negli altri romanzi di Cassieri, si esplicano nella descrizione dell'uomo fermo davanti alla lastra di marmo e dal direttore della biblioteca bloccato nel traffico. Anche se il motivo di stasi è nata da un evento che ha dell'ironico, un uomo aveva frenato all'improvviso perché un gatto nero gli aveva attraversato la strada, Cassieri immette il lettore in una situazione di paradossale dinamicità che porta l'azione ad un punto fermo tale da fissare l'immagine in un'immobilità che avvolge tutta la scena.

Poetica di un infelice è un romanzo in quanto esiste una storia che si sviluppa e la voce narrante accompagna il lettore alla scoperta dell'identità del misterioso personaggio, che appare e scompare ad intermittenza aumentando intorno a sé l'atmosfera di mistero che lo avvolge. Sono i dialoghi sempre lasciati sospesi che trovano continuità e risposta in quelli successivi: nel frattempo il lettore è sempre lasciato con la curiosità di voler sapere di più e viene abbandonato nell'attesa del 
prossimo incontro che avverrà chissà quando. Saranno incontri casuali e in ognuno si apprende qualcosa di nuovo di questo personaggio dall'aspetto e dal comportamento alquanto bizzarri: si impara a conoscerlo nel suo profondo disagio sia intellettivo sia fisico.

Il disagio del personaggio è infatti accentuato dalla sua malattia che lo diminuisce rendendolo diverso e misterioso perché essa incide sulla mente e sul comportamento dell'uomo che lo isola dalla sfera di normali rapporti e di comunicazione con gli altri. Gli attacchi epilettici, che reiterano l'idea di immobilità e di stasi, si presentano in momenti culminanti ad esprimere l'incapacità di interagire e innalzano un muro di incomprensione fra sé e l'interlocutore. Essi diventano paradossalmente un modo di allontanarsi dal mondo al quale sente di non appartenere più. Del resto che il professor Lamanna abbia scelto i versi di Leopardi da ripassare con la vernice rossa è indice di una ribellione repressa e di un'affinità fra sé e il poeta, che si traducono in una forma di isolamento ed incomprensione acuiti dalla loro condizione fisica.

Il malessere e la condizione dell'uomo sono tuttavia paradossalmente accentuati dalla necessità che lui ha di stare con gli altri. Ogni uomo, nel caso specifico, ogni artista, filosofo o studioso, sente la necessità di convogliare le proprie idee che, risultano fallaci e inutili, se dall'altra parte non c'è nessuno a recepirle sulla giusta onda di comprensione. L'assenza di una corrispondenza dove un'idea o una parola trovi un punto di riscontro fa sì che l'intellettuale, come nel caso del professore nel romanzo, non incontrando le sue coordinate di simmetria intellettiva, entra nella dimensione paranoica e avverte l'isolamento come l'esilio dal quale non c'è ritorno, $\mathrm{o}$, in altre parole, la possibilità di riscatto dalla sua infelice condizione. I tentativi di slanci per arrivare alla cognizione di una utopia intellettuale, e quindi il riferimento a Tommaso Campanella, sono contrapposti dal bisogno físiologico e pragmatico nella vita che si 
traducono anche nel bisogno di sonno che Lamanna aveva perduto e della (ri)scoperta del sesso che purtroppo anche qui non trova una corrispondenza simmetrica. Fortunatamente per il professore quando lui sta per commettere un atto di pedofilia arriva un attacco epilettico a bloccarlo. Ed è proprio in questa scena ambientata nel mare della riviera di Ulisse che avrebbe dovuto sortire un effetto curativo alle sue preoccupazioni si scopre un essere sensuale e primordiale. Il lettore trova davanti a sé un uomo che era stato ben anticipato nella sua immagine: "Il sesto senso, o semplice intuizione, mi spingeva a controllare i movimenti di quel fuscello umano col petto ultravilloso, le gambe scheletriche, i piedi affondati nella sabbia, i capelli irti e aggressivi" (Qui è la voce del direttore, 97).

Una volta avvenuto il salvataggio e portato sulla spiaggia dove si era formata un capannello di spettatori, così continua la descrizione dell'uomo:

"Uomini e donne cominciano a diradare con un filo di riluttanza o di ribrezzo. Il "dormiente" mostrava all'aperto il triste quadro clinico: bava verdegialla, occhi sbarrati, pallore preagonico... Comunque era salvo". (99)

Chiaramente il suo stato di salute spiega la condizione della mente e l'immobilità anticipa la sua fine: il capitolo dieci del romanzo è il canto di persona consapevole della propria situazione:

"Ezio, la morte scherza con me, mi lusinga, ha fretta di imbracarmi; e sai come rispondo'Io sono un cardo incommestibile, un fustello primitivo, non ho eredi e sono assuefatto alla sofferenza." (101) 
La voce narrante continua nella rappresentazione di prostrazione profonda in cui l'uomo era caduto:

"Le tesi di laurea sempre più lo stancavano e indispettivano. Finiva per elaborarle come un ciabattino costretto a riparare scarpe posticce di finta pelle. I venditori di tesi annusavano il vento che stava cambiando e contattavano i preferiti di internet capaci di mettere in piedi sessanta pagine di testo in due settimane, oltre la bibliografia riciclata che nessun relatore si sarebbe peritato di passare al setaccio". (101)

Un aspetto importante affrontato da Cassieri è la radice storico-culturale del personaggio, di origine calabrese. Cassieri rende il disagio dell'uomo più profondo scavando nelle sue origini e condizione sociale, l'eredità culturale, il più delle volte scomoda, aumenta la sofferenza del protagonista divenuto capro espiatorio di tutte le confluenze di motivi che ne giustificano la paranoia e pazzia. La provenienza da una regione del Sud oggi, come del resto ieri, continua ad essere un motivo di segregazione. L'accento dell'uomo, la condizione economica della famiglia in cui è cresciuto (continua tuttora l'eterna questione meridionale (12-13)) incidono sul suo carattere e diventano motivi di imbarazzo. Cassieri descrive la storia di una famiglia che non ha possibilità di salvezza e Saverio Lamanna quando arriva nella capitale si rende conto che la sua condizione è riflessa nell'indifferenza della gente, e lui non ha opportunità di riscatto e di redenzione neppure attraverso la sua arte $\mathrm{o}$ capacità: i gesti eclatanti dell'uomo avrebbero dovuto rimuovere i passanti dal loro stato di indifferenza letargica!

Il riferimento alla figura di Giordano Bruno rafforza la funzione che la persona di pensiero dovrebbe avere nella nostra società. Per i tempi che furono Giordano fu un personaggio atipico in quanto aveva esplorato altre 
possibilità per stabilire dei parametri di conoscenza che entravano in conflitto con quello che era non solo la posizione della Chiesa ma anche dei paesi e delle accademie che lo avevano ospitato. Senza dubbio lui aveva anticipato l'intellettuale del Settecento con un ricco bagaglio di conoscenze vecchie e nuove! Ciò potrebbe essere Saverio Lamanna quando esplica la sua creatività nell'ambito dell'insegnamento apportando nuovi metodi in contrasto con i criteri ufficiali. Un innovatore della società con idee soggette agli sbalzi umorali del personaggio, un meteoropatico. T.S.Eliot aveva affermato che per lui il mese d'aprile era il più crudele di tutti i mesi per il cambiamento di stagione che gli causava disturbi emotivi La malattia di Lamanna sortisce lo stesso risultato perché quando lo assale, diventa motivo non solo di malessere fisico, ma anche mentale.

Ad aumentare la condizione di disagio si aggiunge la difficoltà di trovare un lavoro che possa gratificarlo. Di conseguenza la mancata possibilità di esprimersi, di esistere, di vivere si ripercuote nell'assenza di sogni, a discapito di una attività e creatività mentale che viene inibita nei livelli più profondi. "Il trio canonico di memoria, tradizione ed identità" (106) viene ad essere messo in discussione dallo stesso Cassieri perché laddove in Saverio Lamanna diventa causa di scomodo, in molti pensatori oggi è motivo solo di visibilità. I modi e i contenuti che rendono gli uomini visibili sono ciò che opprimono l'uomo e lo rendono infelice, essi diventano la paura di mostrarsi agli altri non solo nella sua miseria fisica, di qui la necessità di essere elusivo ed ambiguo, ma fanno sì che egli desideri rendere invisibile la sua debolezza che lo emargina e che, a sua volta, emargina le sue idee sopraffatte anch'esse dai rampanti opinionisti dai facili consensi per fare solo bella mostra di sé ad ogni costo.

Un processo di involuzione porta all'esigenza di omologare qualunque tipo di attività mentale-culturale impoverendo la capacità di comprensione 
dei livelli più profondi della mente e dell'agire dell'uomo. Quello che viene a mancare è la capacità della dimensione profonda della vita, e la prima espressione dell'uomo a farne le spese è la poesia, di qui la necessità di Saverio Lamanna a ripassare di rosso i versi di Leopardi, cioè la capacità di guardare oltre. Oggi si riesce difficilmente ad andare aldilà della siepe e il tesoro di pensieri custodito nelle biblioteca perdono di valore e significato quando è nelle mani di omologatori, per questo motivo lui sente il bisogno di bruciarle (118). L'assenza delle biblioteche genera la crisi e la visibilità, come l'epilessia del personaggio cassierano:

"Lo stupore del pubblico, e anche un po'di pena mia personale, crescono di tensione quando Saverio elenca i sommi distruttori delle biblioteche: Platone e Borges, Erasmo e Shakespeare, Rebelais e Shaw, Orwell e Gide... Legge nei suoi foglietti il giudizio dello scrittore e drammaturgo Lousie S. Mercier in un'opera del 1781: - ...l'anima si trova affaticata in questa moltitudine di libri che tanto spazio occupano e servono unicamente a turbare la memoria del bibliotecario...". (119)

Il gesto di Lamanna di incendiare la biblioteca di un liceo è un ulteriore sintomo di persona insofferente all'idea che la biblioteca possa essere "[...] tempio disarmato in balia di gente fanatica" (119). E la considerazione "Quel luogo, questo luogo, solo perdendolo riusciremmo forse ad amarlo" (121-2) spiega la spettacolarità del gesto simbolico di Lamanna di dare visibilità ad un mondo che è custode di pensieri che devono uscire allo scoperto, devono essere liberati e, paradossalmente ed ironicamente, gli "strumenti ci sono: qualche passaggio in Tv, qualche dichiarazione gridata in un convegno, la denuncia dei propri affanni .... Il grimaldello è unico per tutti: Vi-si-bi-li-tà" (130). 
Un personaggio così concepito fatica a vivere nei tempi di oggi dove la comunicazione si basa sull'immediatezza sinottica e per la stessa ragione, chi vive la vita ancorata a canoni di comunicazione diversi, non riesce a sopravvivere come avviene per Bartleby, lo scrivano nel racconto di Melville.

\section{Opere consultate e citate}

Cassieri, Giuseppe

Lepeneis, Wolf

Melville, Herman
2008 Poetica di un infelice. Lecce: Manni Editore, 2008-10-26.

1999 Il muro di Gutenberg. Venezia: Marsilio.

1998 Ascesa e declino degli intellettuali in Europa. Roma - Bari: Laterza, La caduta degli intellettuali nell'occidente.

2008 Bartleby, lo scrivano. Roma: Gruppo Editoriale L'Espresso SpA. 